\title{
Conflictos bioéticos en el paciente con problemas de salud mental
}

[Cómo citar este artículo: Cornejo, F., Mejía, D. Conflictos bioéticos en el paciente con problemas de salud mental. Práctica Familiar Rural. 2019 noviembre; 4(3).]

\section{Fernando Cornejo León [a], Daniel Mejía Peñafiel [b]}

a. Médico Psiquiatra, Neuropsicofarmacólogo, Director del Postgrado de Psiquiatría de la Universidad UTE. b. Abogado, Especialista en Gestión Pública y Abogacía del Estado, Universidad UTE.

DOI: https://doi.org/10.23936/pfr.v4i3.136

Recibido: 20/09/2019 Aprobado: 20/11/2019

\section{Resumen}

Con este articulo se pretende abrir el debate en nuestro medio, sobre los principios bioéticos en los pacientes con problemas graves de salud mental que no están en capacidad de discernimiento de sus decisiones. A partir de un caso clínico y su evolución iremos analizando los alcances médicos y bioéticos, y su implicancia en el ejercicio clínico por parte de quienes trabajan en los equipos de salud mental. Trataremos de dar un par de conclusiones que permitan tratar el tema, en una sociedad en la que la salud mental, es aún un tabú.

Palabras clave: salud mental, principios bioéticos, legislación

Bioethical conflicts in the patient with mental health problems

\section{Abstract}

The present brief communication, intends to open the debate in our environment, about bioethical principles in patients with serious mental health problems who are not able to discern their decisions. From a clinical case and its evolution we will analyze the medical and bioethical scopes, and their implication in the clinical exercise by those who work in mental health teams. We will try to give a couple of conclusions that allow us to address the issue, in a society where mental health is still a taboo.

Keywords: mental health, bioethical principles, legislation 


\section{Introducción}

El paso del origen mágico o sobrenatural de los síntomas psiquiátricos hacia una explicación que denote su origen orgánico, no solo ha tomado siglos enteros, sino que todavía se abre paso entre las creencias populares.

Cuando la enfermedad mental pasa a clasificarse como una patología de origen biológico, se piensa que la misma debería reunir todos los requisitos de una enfermedad clínica, es decir signo-sintomatología, un correlato clínico que lleve a un diagnóstico y un pronóstico derivado de la intervención, situación que se confirgura cada vez con más firmeza, pero con menos exactitud de lo que un sistema positivista requiere.

No son pocas las vertientes teóricas contradictorias al positivismo biologista, así conviven en un mismo lugar la psiquiatría biológica, el psicoanálisis, las teorías de la conducta y el aprendizaje, los postulados sistémicos y la propia antipsiquiatría.

Dentro de este marco, los psiquiatras que ejercen la práctica clínica, el Sistema Nacional de Salud y el sistema de justicia deben atender casos cada vez más frecuentes de pacientes que no están en condiciones de tomar sus propias decisiones, siendo un riesgo para sí mismo y para terceros, pero los cuales, no están preparados.

2. Caso clínico

N.N es una paciente femenina de 25 años de edad, soltera, hija única, huérfana de padre desde los 16 años. Presenta antecedentes de tratamientos psiquiátricos por desórdenes de la condutca no especificados y con toma de medicación (antipsicóticos) esporádica desde los 13 años, también asistió a terapia psicológica en repetidas ocasiones, la cual es abandonada.

En la actualidad vive con la abuela materna, abandonó los estudios secundarios en varias ocasiones y en este momento cursa en un colegio a distancia sin resultados claros; en diversas ocasiones no duerme en su casa y ha sido detenida por la policía en un par de ocasiones por presentar conductas agresivas en estado de embriaguez.
Acude a la consulta psiquiátrica de segundo nivel de atención por presentar alucinaciones visuales, auditivas, ideas delirantes de predominio mísitico y conducta desorganizada de predominio agresivo.

La paciente reconoce haber consumido sustancias psicotrópicas, cocaína, ayahuasca, metaanfetaminas y otras no determinadas en los últimos tres meses además de consumo diario de marihuana.

Como medida emergente se inicia con antipsicóticos típicos por vía oral a dosis estándar de adulto y benzodiazepinas, se solicita un panel de drogras, imagen por resonancia magnética cerebral (IRM) y eletroencefalograma.

Acuden a la consulta de control, dos días después, se encuentra una IRM dentro de los límites de la normalidad, en el electroencefalograma hay descargas esporádicas frontales y temporales con tendencia a la generalización secundaria, en el panel de drogas se encuentra positivo para cocaína, marihuana y benzodiazepinas (estas últimas habían sido prescritas en la primera consulta).

La abuela refiere que se incrementaron los episodios de auto y heteroagresión, generó destrozos en su casa, dice que las alucinaciones han incrementado, en el examen semiológico se objetivan alteraciones sensoperceptivas y hay mucha agresividad. Se le administra una benzodiazepina por vía oral en la consulta ambulatoria y se lo deriva al servicio de emergencias psiquiátricas para internación por riesgo para sí y para terceros.

\section{La emergencia psiquiátrica: el principio de beneficiencia}

El principio de beneficencia, que definido de manera muy simple se reduce a "hacer el bien", aplicando de manera adecuada, todos los procedimientos clínicos, diagnósticos y terapéuticos iniciales en un paciente, que como el caso descrito genera un riesgo pleno para sí y para terceros.

La urgencia y la emergecia psiquiátrica, momento en el cual, el juicio del paciente, es decir la capacidad de comparar, relacionar, identificar y valorar las situaciones que están a su alrededor, está alterada, ameritan una acción por parte del personal sanitario, sin que necesariamente medie la aceptación explícita del paciente. 
El juicio desviado, por definición, genera incapacidad en la toma de decisiónes fundamentales y jurídicamente, la demostración de la existencia del mismo, podría generar inimputabilidad ante un delito comentido durante la permanencia de la desviación del juicio.

Es una obligación del personal de salud informar de manera suficiente, oportuna y amplia sobre la necesidad de la provisión del beneficio, y la utlidad que tiene el mismo en balance con una intervención para el paciente y para terceros, incluyendo el núcleo más cercano.

Se debe entender que si aplicarmos el principio de beneficencia, sobre todo beneficencia activa, en este tipo de pacientes, protegeremos la integridad del enfermo y los derechos de terceros y al menos logramemos tener un control de daños que el propio proceso psicopatológico puede llegar a ocasionar.

En el caso descrito, la acción del profesional de la salud es obligatoria y la omisión podría ser punible, quedando claro que es un principio adopatar todos los medios posibles para que, de ser necesaria la internación, se deben agotar todos los esfuerzos para que la misma se haga de manera voluntaria.

Probablemente lo más discutido en estos casos sea el de la internación no voluntaria, que necesariamente deberá cumplir con la evidencia de que el trastorno mental implique riesgo para sí o para terceros y la semiología psiquiátrica demuestre que no existe la capacidad de toma de decisiones (juicio desviado).

El juicio podría estar desviado, sea porque (como en el caso) existen alteraciones graves de la sensopercepción que le llevan a perder casi por completo la capacidad de comparar, relacionar, identificar o valorar el para poder llegar a una conclusión, o porque la esfera afectiva, llega a tener grados de daño que perturban la capacidad de valorar.

Debemos aclarar que médicamente, en este caso estamos evaluando el discernimiento de la persona, es decir la integridad que tienen los niveles individuales de funcionamiento de su sistema nervioso para tomar decisiones sobre si, y no la capacidad del individuo que es un tema jurídico.

PRÁCTICA FAMILIAR RURAL| Vol.4| No.3| Noviembre 2019 | www.practicafamiliarrural.org
4. La internación breve: el principio de la no maleficencia

El tiempo de las internaciones prolongadas en las que se sacaba al enfermo mental de su medio y era más complejo reintegrarlo que integrarlo es un tema del pasado, pero vive en el imnaginario colectivo. En la actualidad, la evidencia dice que la internación corta (menos de 28 días), no tienen mayores reingresos hospitalarios y no abandonan el tratamiento más que los pacientes que han sido dados de alta después de una internación prolongada.

En un paciente internado por emergencias, el objetivo clínico principal, será diagnosticar, re-evaluar el diagnóstico (en caso de haber sido realizado antes), ajustar dosis de medicación, en fin estabilizar al paciente para que recupere sus capacidades plenas y pueda reintegrarse a la sociededad, con el adecuado manejo y seguimiento ambulatorio.

Cuando hablamos de internaciones voluntarias, por regla general, nos referimos al momento en que se están recuperando las capacidades de adaptación, sea desde la relación sensopercepción-pensamiento-juicio o, en su defecto, desde el ámbito afectividad-pensamiento-juicio, las determinaciones clínicas van en concordancia con la recuperación del paciente y la del medio que lo rodea para dar un alta para continuación por tratamiento ambulatorio.

En este tipo de casos, no solemos encontrar con otro tipo de problemas, relacionados principalmente, con las incapacidades del medio, principalmente el famililar, para generar la acogida y cuidados necesarios, sobre todo cuando hablamos de enfermedad crónica.

Queda claro, sin embargo, que la internación prolongada, no es lo más eficiente, no genera beneficios desde lo clínico y trae pérdida de oportunidades en el propio enfermo, mantener internaciones prolongadas, la famosa "institucionalización", violaría el principio de no maleficencia.

\section{Tratamiento involuntario: muchas interrogantes}

Hay largas experiencias sobre el uso de tratamiento involuntario en régimenes políticos totalitarios como la dictatura en Argentina en 1976 o la de Chile en 1973, con el fin de acallar a opositores políticos. Todo estado nacional, dentro de sus principios normativos constitucionales y legales, debe tener claridad sobre los límites del tratamiento médico involuntario. 
Sin embargo, varios instrumentos de derechos humanos aceptan como los Principios EM (1991), el Convenio Europeo para la Protección de los Derechos Humanos y la Libertades Fundamentales (1950) y la Declaración de Hawaii (1983), entre otros incluyen la necesidad de la existencia de tratamientos involuntarios cuando el paciente carezca de capacidades de discernimiento para tomar su propia decisión.

Para el caso referido, deberemos partir de situaciones básicas, pero que no deben dejar de ser mencionadas, como el trato respetuoso a la dignidad humana, la calidad de las unidades asistenciales y de su personal y que, durante toda la evolución del caso, el paciente esté en pleno conocimiento sobre cuáles son los procedimientos que se están practicando con el fin de mejorar su situación y para que el tratamiento en sí, se vuelva voluntario.

Es fundamental señalar, que más allá del aspecto meramente ético, es necesaria la existencia de normativa estatal detallada a este respecto, con el fin evitar excesos y que las autoridades sanitarias tengan los debidos mecanismos de control y sanción de ser del caso hacia instituciones de salud y los mismos profesionales.

En este caso si debemos ser enfáticos en que las personas solo podrán ser tratados de manera involuntaria dentro de una institución psiquiátrica, cuando el profesional calificado determine que existe riesgo de daño inminente para sí o para terceros, debido a la enfermedad mental concurrente, y que el paciente no está en capacidad para comprender que debe ser internado y tratado o en su defecto la no retención en la institución puede generar un peor pronóstico. Siempre deberá ser preferible la participación de dos profesionales y adicionalmente, si es que existe una retención prolongada del paciente, la institución deberá estar facultada para ello por parte de las autoridades del sistema de justicia o pretección.

Una cuestión fundamental para los clínicos que leen este artículo, es que siempre, el plan de tratamiento o sus objetivos, deberá estar determinado previamente, al inicio del tratamiento en contra de la voluntad y si existe alguna modificación, deberían ser puestos en consideración de un ente revisor independiente, o por lo menos de otro colega independiente.

Desde el punto de vista ético, cabe una pregunta adicional, sea por cualquier tipo de presión que exista, ¿es lícito que el profesional de la salud interne por solicitud de los familiares y sin que el paciente tenga noción alguna del por qué de la internación y la información sobre cuál es el plan de la misma? En este primer caso se violenta de manera extrema el principio de la autonomía situación que es vista muy a menudo en la prensa de nuestro país, sobre todo en los casos de clínicas que tratan adicciones y patologías duales con las co-terepeutas como facilitadores del proceso.

Pero, por otro lado, tampoco se puede reducir el tema a una excesiva autonomía del paciente, que, con fallas en el discernimiento, toma la decisión de dejar una medida extrema de internamiento y tratamiento involuntario, poniéndose en riesgo y también poniendo en serias dificultades a su familia y a la sociedad.

Una vez más, el rol del clínico es fundamental para la resolución de este tipo de conflictos y la participación activa del Estado, con normativa clara y detallada, ayudará al clínico para que no haya el menor número de dificultades posteriores, para una serie de fenómenos cuyos resultados no son predecibles.

\section{Evolución y resolución del caso}

La paciente N.N. fue ingresada y recibió antipsicóticos incisivos y benzodiazepinas por vía parenteral debido a una agitación psicomotriz al ingreso, fue debidamente controlada en sus signos vitales, hidratación e higiene mientras permaneción sedada.

A las 24 horas mantuvo una entrevista con el psiquiatra, teniendo conductas extremadamente manipuladoras, con tendencia a negociar el tratamiento. En el examen semiológico se evidenció la presencia de alucinaciones sensoperceptivas de tipo visual, auditivo y cenestésicas, pero que la paciente utilizaba para manipular para recibir más medicación.

Por el antecedente de los resultados del electroencefalograma, el servicio de neurología recomienda la administración de ácido valproico 1 gr. al día, en conjunto con las indicaciones del psiquiatra que fue el mantener antipsicóticos incisivos por vía oral y benzodiazepinas también por vía oral para evitar el síndrome de abstinencia.

La paciente permanece internada, pero con dos intentos de fuga, también se encuentra que en su habitación reunía hojas de plantas ornamentales con el fin de aspirarlas, y que intentó agredir a otros pacientes con el fin de obtener cigarrillos.

Las pruebas psicométricas, identificaron que había rasgos antisociales en la personalidad. 
A las dos semanas de internación existía algo más de conciencia de enfermedad, se realizan dos sesiones de terapia familliar, en las cuales se indica la necesidad de un dispositivo para el alta que implique un cuidado contínuo, actividades laborales controladas permanentes y tratamiento ambulatorio por psiquiatría, neurología y psicología.

La familia tenía dos opiniones, por un lado, el mantener una internación mucho más prolongada y por otro trabajar el alta sin dispositivo terapéutico. El médico y la institución plantean que lo mejor, hasta que exista un acuerdo que beneficie al paciente, es no indicar el alta de manera precoz, pero la familia y la paciente, deciden un alta en contra de opinión médica.

En este caso, la familia, y el propio vacío en la normativa vigente, dio prioridad al principio de autonomía, aún cuando se explicaron los riesgos de que una externación precoz y sin condiciones adecuadas, llevaría a perpetuar la enfermedad. De esta manera, el tratamiento mitigó de manera limitada, cierta sintomatología clínica muy florida y se imposibilitó el manejo de otros aspectos como prevención, promoción y rehabilitación.

Es imposible decir el dar una regla general sobre la primacia de un principio bioético sobre otro en salud mental, pero definitivamente, se debe tener protocolizado, de mejor manera este tipo de situaciones.

\section{Conclusiones para abrir el debate en el Ecuador}

Los trastornos mentales, en los cuales está afectada la capacidad de discernimiento, requieren de un conocimiento claro de la normativa vigente en el país y sobre los principios bioéticos a aplicarse con el fin de salvaguardar el bienestar del paciente y de terceros.

En las urgencias y emergencias psiquiátricas es indispensable la aplicación del principcio de beneficencia, específicamente, de beneficencia activa con el fin de proteger al individuo.

Las internaciones prolongadas, no han demostrado ser más eficientes que las no prolongadas, por tanto, las primeras podrían estar violentando el principio de no maleficencia.
El principio de autonomía no es totalmente aplicable para los pacientes que no tienen total capacidad de discernimineto, pero tampoco es factible cortarlo como se ha observado en muchos centros de tratamiento ecuatorianos.

El principio de autonomía no puede irse sobre el principio de justicia, ante una persona que requiere cuidados por parte del sistema de salud.

Se requiere normativa clara y extensa, que permita cumplir los tratados internacionales, la constitución y evitar excesos.

\section{Referencias bibliográficas}

Babalola O, Gormez V, Alwan NA, Johnstone P, Sampson S. Length of hospitalisation for people with severe mental illness. Cochrane Database of Systematic Reviews 2014, Issue 1. Art. No.: CD000384. DOI: 10.1002/14651858.CD000384.pub3 Organización Mundial de la Salud. Manual de recursos sobre salud mental, derechos humanos y legislación de la OMS. 1. ISBN 9243562827 ISBN 9789243562827 (Clasificación NLM: WM 2006. Megia Ma., Moreno J., Salud mental y Bioética: Reflexiones desde una perspectriva multidisciplinar, Generalitat. Conselleria de Sanitat, 2013 Ramos Montes J, "La competencia mental y el internamiento involuntario en psiquiatría: entre el derecho y la bioética”, Labor hospitalaria, no 264, 2, 2002, p. 103 Beauchamp, T. L., \& Childress, J. F. (2008). Principles of Biomedical Ethics (6a ed.). Nueva York:

Oxford

Gracia, D. (1989). Fundamentos de bioética. Madrid: Eudema. 